\title{
Recent Advances in Integrated Photonic Jet-Based Photonics
}

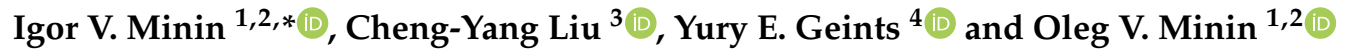 \\ Physical Department, Tomsk State University, 30 Lenin Avenue, 634050 Tomsk, Russia; ovminin@tpu.ru \\ 2 School of Nondestructive Testing, Tomsk Polytechnic University, 36 Lenin Avenue, 634050 Tomsk, Russia \\ 3 Department of Biomedical Engineering, National Yang-Ming University, Taipei City 11221, Taiwan; \\ cyliu66@ym.edu.tw \\ 4 V.E. Zuev Institute of Atmospheric Optics SB RAS, 1 Zuev Square, 634021 Tomsk, Russia; ygeints@iao.ru \\ * Correspondence: IVMinin@tpu.ru
}

Received: 25 April 2020; Accepted: 10 June 2020; Published: 11 June 2020

check for updates

\begin{abstract}
The study of accelerating Airy-family beams has made significant progress, not only in terms of numerical and experimental investigations, but also in conjunction with many potential applications. However, the curvature of such beams (and hence their acceleration) is usually greater than the wavelength. Relatively recently, a new type of localized wave beams with subwavelength curvature, called photonic hooks, was discovered. This paper briefly reviews the substantial literature concerning photonic jet and photonic hook phenomena, based on the photonic jet principle. Meanwhile, the photonic jet ensemble can be produced by optical wave diffraction at 2D phase diffraction gratings. The guidelines of jets' efficient manipulation, through the variation of both the shape and spatial period of diffraction grating rulings, are considered. Amazingly, the mesoscale dielectric Janus particle, with broken shape or refractive index symmetry, is used to generate the curved photonic jet-a photonic hook-emerging from its shadow-side surface. Using the photonic hook, the resolution of optical scanning systems can be improved to develop optomechanical tweezers for moving nanoparticles, cells, bacteria and viruses along curved paths and around transparent obstacles. These unique properties of photonic jets and hooks combine to afford important applications for low-loss waveguiding, subdiffraction-resolution nanopatterning and nanolithography.
\end{abstract}

Keywords: photonic jet; photonic hook; microparticle; cuboid; diffraction grating

\section{Introduction}

Nowadays, bending light beams are being intensively investigated for propagation in free space. The most well-known is the Airy beam, which was first predicted by Berry and Balazs in 1979 [1] and experimentally demonstrated by Siviloglou et al. in 2007 [2]. Since that time, different types of self-accelerating photonic beams [3-6] have been proposed in a broad range of applied science-related fields, including trapping particles [7], plasma [8], surface plasmon polaritons [9,10], non-lineal optical trapping [11] and electron beam trapping [12]. One of the most important applications of the Airy beams is the development of optical tweezers for moving micron-sized particles along curved paths, which allows one to reduce the light loading on biological tissues. The long-focus Airy beam contains the maximum concentration of the light energy, similar to the focal plane of the Gaussian beam. According to the beam theory [13], the main parameter of such beams is the propagation length along the curved path (acceleration). A common optical scheme for Airy beam formation includes a cylindrical lens and a spatial light modulator (SLM), which operates only at restricted power. The latter is mounted in the front focal plane of the cylindrical lens, while the Airy beam generates in its back focal plane and nearby. The cubic phase modulation of an incident Gaussian beam generated by a modulator 
acts as a cubic lens, whereas the curved path is determined by the acceleration rate of the cubic phase change [2]. The Airy laser beams are generated by binary phase diffractive optical elements [13]. The phase diffraction gratings are used as SLM analogs, which create bending diffraction orders [14,15]. The metasurfaces are also used for generating the Airy beams; however, the manufacturing process of the metasurfaces is rather complicated and expensive [16].

The diffraction-free pulsed beams in space-time have been the subject of many interesting studies [13]. The lightwave packet becomes a sort of non-paraxial Airy-pulsed beam. Such optical pulsed beams with self-bending parabolic spatial profiles propagate in the forward direction [17]. The acceleration-free Airy wave packet is synthesized by changing its spatiotemporal spectrum. On the other hand, the Bessel beam emitted from expanding circles on the input plane propagates along straight trajectories [18]. In the following studies, it is possible to produce Bessel-like beams along spiral, snake or zigzag trajectories [19-21]. An advantage of the Bessel-like beams is that the convex trajectories are not necessary. Furthermore, abruptly autofocusing beams are generated by Fourier-transforming an apodized Bessel beam [22]. This can be used as a robust photophoretic trap for airborne particles. Three-dimensional manipulation of the trapped particles is experimentally illustrated by applying vortex Bessel-like beams. The trapped particles follow the spiral, parabolic and hyperbolic trajectories along the high-intensity ring of the high-order Bessel beam [23]. The Airy beam innately provides higher contrast and enlarges the field of view. The characteristic asymmetric excitation pattern of the Airy beam can be applied to a step change for light-sheet microscopy [24,25].

It is important that the diameter of the Airy beams is usually several incident wavelengths, equaling the diameter of an optical element which is much larger than the wavelength [13]. Scaling the Airy beam generation from visible light to the terahertz range is not always possible. This is because SLMs cannot operate in the terahertz range, due to the absence of materials with the required modulation [26]. Moreover, the main lobe of a finite energy Airy beam is not observed directly behind the cubic phase element and transition region, where the initial intensity distribution of the incoming beam is transformed into the distinct Airy pattern [27]. With the aim of encouraging photonic study, we summarize the progress made in the photonic area of the jet and hook to-date. The structures of a localized field for a photonic jet and hook differ fundamentally from Airy beams [13]. Moreover, the curvature of the Airy-like beams is determined by a parameter that determines the rate of rise of the cubic phase [13], and is usually greater than several wavelengths. The results and discussions in this paper include the initial identification of the photonic jet and hook for dielectric microparticles, subwavelength curved localized light beams, photonic jet array by diffraction grating, experimental observations, and the potential applications.

\section{Near-Field Curved Beams}

It is well known that when diffraction arises from the dielectric edge of the Fresnel zone plate, the edge wave has the eikonal approximation $\left(S \sim Z+X^{2} / 2 Z\right)$, where $X$ is the transverse coordinate and $Z$ is the longitudinal coordinate [28]. A solution of the diffraction at the edge of the semi-infinite opaque screen is a paraxial two-dimensional light, for which the argument for the complex amplitude function depends on variables ( $X^{2} / Z$ ) [29]. Instead of the Airy beams, the optical beam has a parabolic path $y=x^{2}$, whereas the considered edge beams in the equation propagate along a root-parabolic path $y=\operatorname{sqrt}(x)$. Figure 1 shows the full-wave simulation results of the diffraction on a rectangular phase plate, under the illumination of a linearly polarized plane wave. It can be seen in Figure 1a that the curvilinear field localization areas arise in the region of the plate edge. As the plate width decreases, these localization regions approach each other. In cases of a small plate width, these optical beams begin to interfere with each other, and form a central classical photonic jet during diffraction via a phase step $[30,31]$. It should be noted that such curved beams can have the self-healing property of the Airy beams, as shown in Figure 1d. 


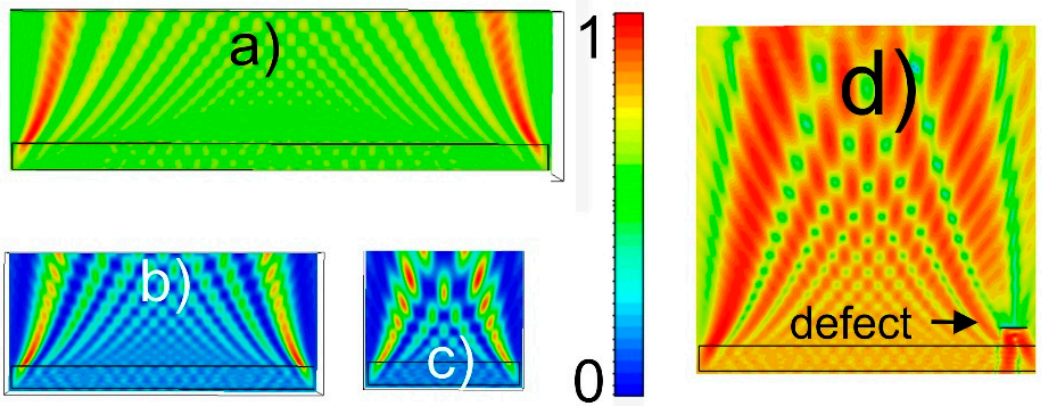

Figure 1. Full-wave simulation results of the diffraction on a rectangular phase plate, with widths of (a) $36 \lambda$, (b) $24 \lambda$, (c) $12 \lambda$, and (d) a rectangular phase plate with defect.

In this paper, we consider the mesoscale particles for the generation of photonic jets. The Mie parameter $(q=2 \pi \mathrm{r} / \lambda)$ is used to determine the particle size. Between the nano-scale optics $(q \sim 1)$ and traditional optics $(q>100)$, there is an intermediate range $(q \sim 10 \ldots 40)$ of the particle size in particular. In the visible light region, this intermediate range matches the micron-sized particles (from several micrometers to tens of micrometers). The photonic nanojets (PNJs) are earlier detected by using the particles in this intermediate range [32-34]. The generation of bounded light beams in the near-field is crucial for many applications, for example, for optical memory systems. A subwavelength effect (PNJ), created by microspheres and microcylinders, can be used in Raman spectroscopy [35] and nanolithography [36]. Moreover, the optical properties of the PNJ can be changed by the interaction between the nanojet and nanoparticle, and the amplification of back-scattered amplitude from the microparticle is increased by several orders of magnitude [34]. This opens new perspectives for designing sensors of high space resolution, capable of recording objects whose size is hundreds of times smaller than that of sensors.

The PNJ phenomenon is very attractive due to the simplicity of its implementation and the compact size of the focusing particle. On the other hand, the minimum beam width of the PNJ is about $\lambda / 3[34,37,38]$, where $\lambda$ is the wavelength of the incident light. Thus, it is necessary to search for new methods to further reduce the size of the focal spot of the PNJ. For deep subwavelength-scale focusing, beyond the solid immersion diffraction limit of $\lambda / 2 n$, a nanohole-structured dielectric microsphere was proposed for strong light confinement [39]. The field enhancement is due to the permittivity contrast between the nanohole material and the dielectric microparticle material. The proposed mesoscale nanostructured sphere has several unique properties. For example, it could produce a high optical power and electric field intensity in low-index hole materials (air), whereas this cannot be achieved through a conventional PNJ produced by spheres, without a nanostructure and with the same diameter [34]. The incident light wave is confined by the dielectric particle inside the nanohole, even when the hole diameter is deeply sub-wavelength (at least $\lambda / 40$ ). The PNJ resolution near the shadow surface of the particle is comparable to the nanohole size (beyond the solid immersion diffraction limit).

Figure 2 shows the example of field localization in a mesoscale nanohole structured sphere. The manufacturing of a nanohole on the rear surface of a dielectric mesoscale particle allows us to compress the field localization characteristic of the PNJ to this nanohole size. The electromagnetic fields near the edges of the nanohole are large, due to the associated edge singularities. In addition, these singularities naturally combine energy into all higher-order modes. Because most higher-order modes are evanescent and contribute only to the reactive field of this emitter, they may not propagate in the far field. Therefore, the higher-order modes are present in the near-field of the particle. By designing the nanohole accordingly, it is possible to direct the output power flow near this nanohole to the desired optical beams, i.e., higher-order modes can be used to control the directivity of radiation in the near-field. The mesoscale nanostructured dielectric particles provide better field localization, even in the case of the resonant excitation of an ordinary spherical particle, for which the transverse 
size of the radiation localization region can reach $\lambda / 5$ with a giant enhancement of the magnetic field component $[40,41]$.
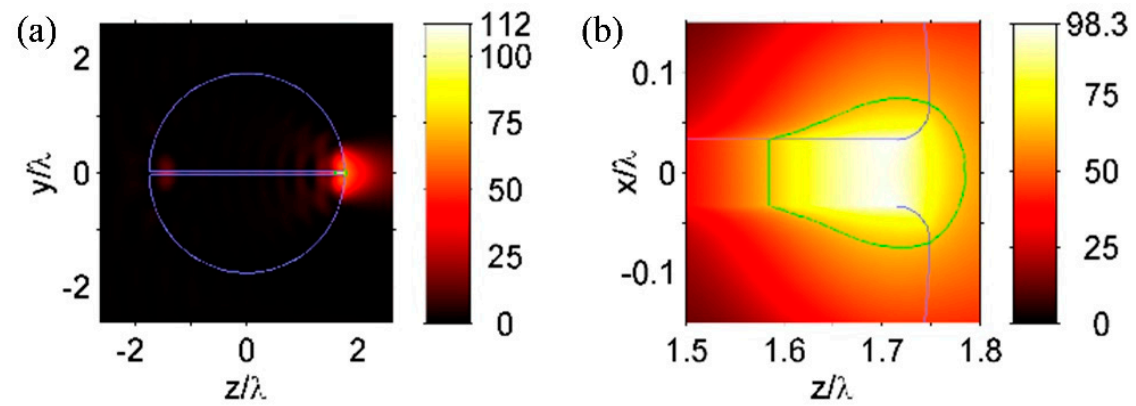

Figure 2. Field localization by nanostructured spherical particle at $d=\lambda / 15$ in (a) $z y$ plane and (b) $z x$ plane [36].

\section{Subwavelength Curved Localized Light Beams}

Recently, a new type of self-bending light beams called a photonic hook ( $\mathrm{PH})$ has been reported [42]. These photonic hooks possess unique properties, such as extremely low curvature (less than the emission wavelength), extreme acceleration, a propagation length of several wavelengths and the minimum width of the beam waist [43]. An ordinary mesoscale optical solid-state dielectric Janus particle with broken symmetry is used to generate the photonic hook near to its shaded surface. Using the photonic hook, one can not only improve the resolution of optical scanning systems, but also develop optomechanical tweezers for moving micron-sized particles, cells, bacteria and viruses, along curved paths and around transparent obstacles via continuous-wave [44] or pulsed [45] illumination. At the same time, the approach based on the interaction between a plane wave and an asymmetric dielectric particle can be scaled to another spectrum range. It was experimentally demonstrated in the terahertz range [46], in acoustics [47], and predicted for surface plasmon [48], recently experimentally confirmed in [49], as shown in Figure 3. The time of the full-phase oscillations of the optical wave changes due to the asymmetric shape of the mesoscale particle. As a result, we have a bent output beam of light. In particular, this particle shape is first considered in [37], and combines prism refraction with cube diffraction.

(a)

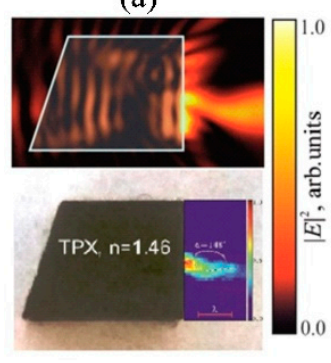

(b)

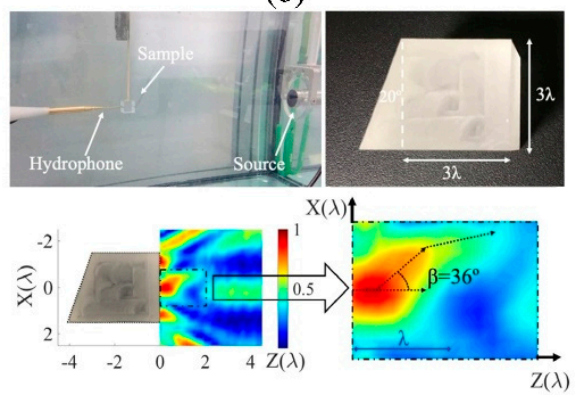

(c)

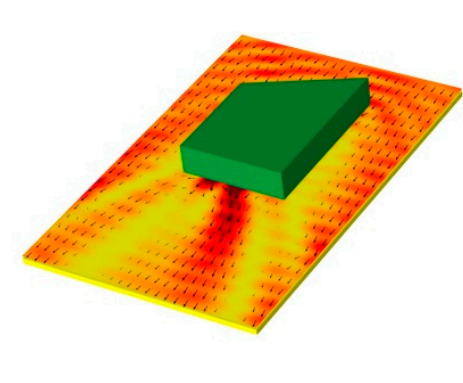

Figure 3. Photonic hook based on Janus particle: (a) THz [44], (b) acoustic [45] and (c) plasmonic [46].

Figure 4 shows several methods for forming photonic hooks. In [50], the distortion of the particle's spherical aberration was suggested, via placing a cube-shaped particle with a smaller size and another refractive index inside the spherical particle, in order to form a photonic hook. However, this approach is not expedient because of substantial difficulties in its implementation. Figure 4 a shows the simulation for a multifocal curved beam based on an $\mathrm{SiO}_{2}$ microsphere with a diameter of $433 \lambda$ (which does not meet the mesoscale condition, and is beyond the range of PNJ existence) in the optical waveband. By adjusting the relative position between an off-axis Gaussian beam and a spherical particle, it is 
possible to control the beam curvature [51]. A photonic hook, upon asymmetric illumination of a mesoscale cylinder with a mask, is shown in Figure 4b [52]. Furthermore, the double photonic hooks are generated by a specially designed five-layer dielectric cylinder [53]. Furthermore, the dielectric symmetry-broken particles with different refractive indexes were proposed and numerically studied for the generation of photonic hooks [54-56] (Figure 4c). At the same time, single plane-wave illumination and twin-ellipse microcylinders were numerically studied to produce twin photonic hooks [57].

In order to differentiate between cells, a concept for examining the in-vitro biomedical application is proposed, to guide the cells in a curved trajectory [58]. However, this substrate generates its own scattered field, and the field of the photonic hook is destroyed in this geometry due to the interference effect. With the use of the near-field, self-accelerating photon beams of the hook type, it is important to develop methods of control for their parameters, including particles of different types and the methods of the photonic hook formation. It is also important to develop a method of property control for generating self-accelerating light beams, and this can be used in different scientific and application areas, including portable telecommunication systems. Thus, the change in the curvature (e.g., due to polarization or a dynamic change in the incident radiation and refractive index) provides a control for the beam position in space, its acceleration and self-reconstruction.

The main idea of curved photonic flux (photonic hook) generation by means of an index-contrast Janus particle is elucidated in Figure 5. According to previous references [12,13], the optical wave should acquire an asymmetric phase during propagation through a particle for the generating of a photonic hook. It can be achieved in two ways, either by means of a particle with shape asymmetry, or by a particle with built-in refractive index asymmetry $\left(n_{2} / n_{3}\right)[53,55,59]$, as shown in Figure 5a. Thus, the desired phase modulations can be acquired using the gradient distribution of microparticle material, e.g., by merging two materials with different optical properties. The detailed analysis of the energy flow structure near a Janus particle shows [59] that the curved photonic jet is formed mainly by the two most intensive optical fluxes (marked $S_{1}$ and $S_{2}$ in Figure 5c) emerging in the upper and lower parts of the particle. Due to wave diffraction on rectangular facets, these fluxes are always directed at an angle to each other, and their superposition forms a leaky external optical field in the form of a tilted photonic jet. The optical contrast between the halves of a Janus particle leads to flow imbalance and energy redistribution in favor of the flux passing through the lower refractive index part, according to Snell's law. As a result, the external photonic jet first acquires a refractive slope towards the exit face of the particle, and then bends due to interference of the fields in two counter-flows. Moreover, depending on the ratio of the Poynting vector flux's strength, the photonic hook can substantially change its bending angle $\alpha_{h}$. The bending angle dependencies of the photonic hook on the index contrast $\left(n_{2} / n_{3}\right)$ of the Janus particle and its edge inclination angle $\gamma$ are shown in Figure $5 \mathrm{~d}$,e. It is clearly seen that in both cases there is the specific range of optical contrasts-1.01 $<n_{2} / n_{3}<1.12$-when photonic flux bending is most pronounced. The increase of inclination angle $\gamma$ of the secant plane, dividing the Janus particle into two parts, causes the increase of the wave refraction angle at the interface between the parts, thus introducing an imbalance between the light energy fluxes. Mostly, this affects the exit angle of the photonic beam outside the particle, whereas the bending angle of the right arm of the photonic hook is influenced to a lesser extent. The highest degree of photon hook bending is achieved in the case of a bar divided exactly along its diagonal. 

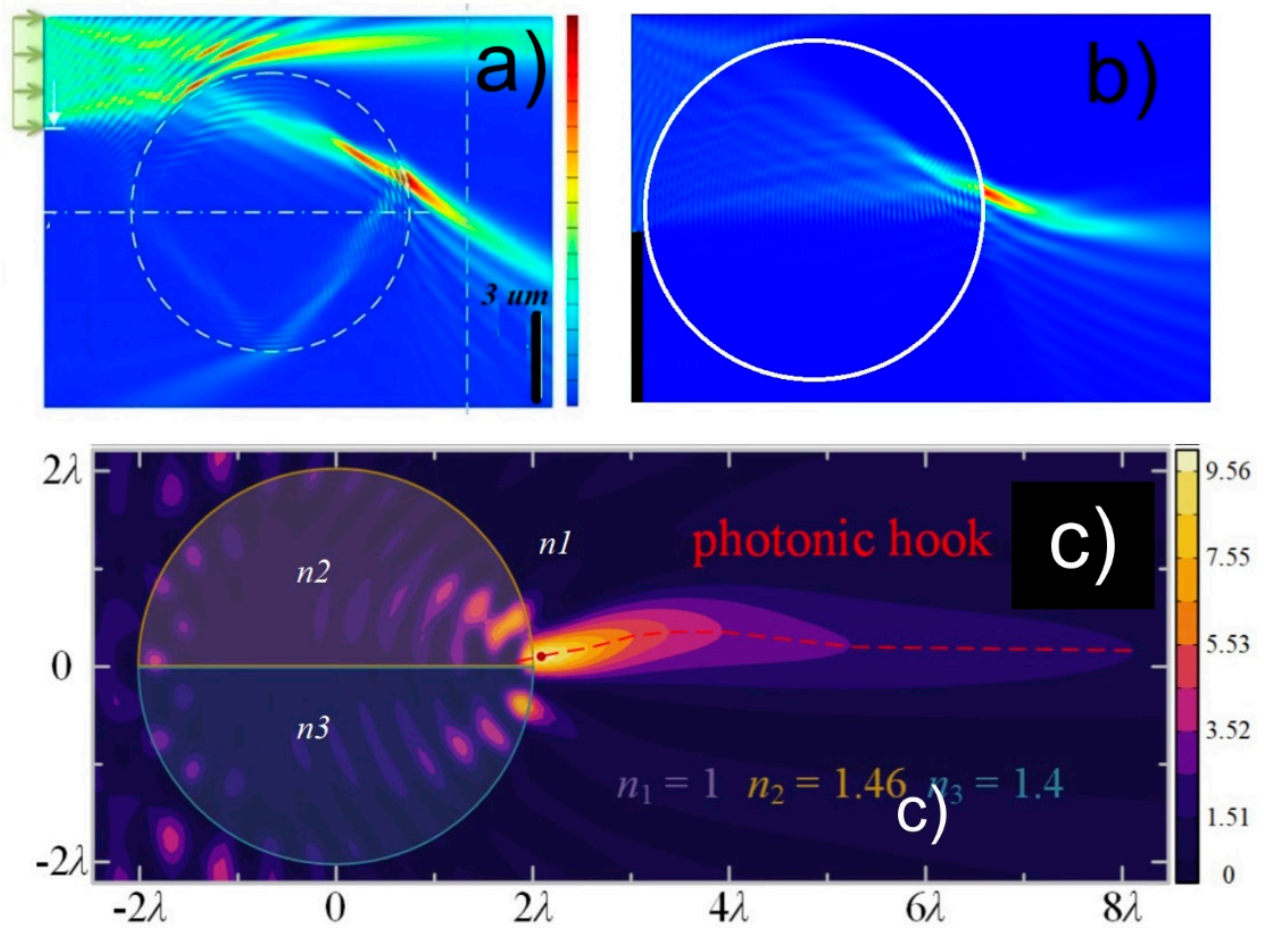

Figure 4. Photonic hook based on Janus particle: (a) cylinder with off-axis Gaussian beam [51], (b) cylinder with partially broken wavefront [52], (c) cylinder with two materials [56].

(a)
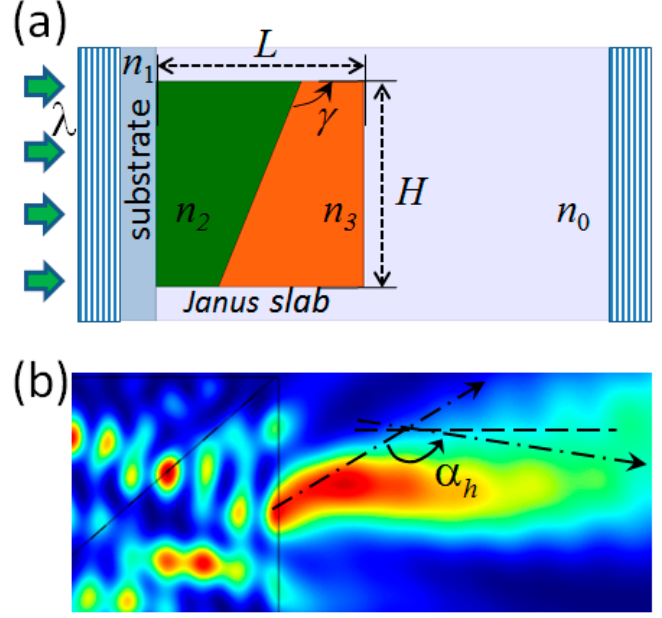

(c)

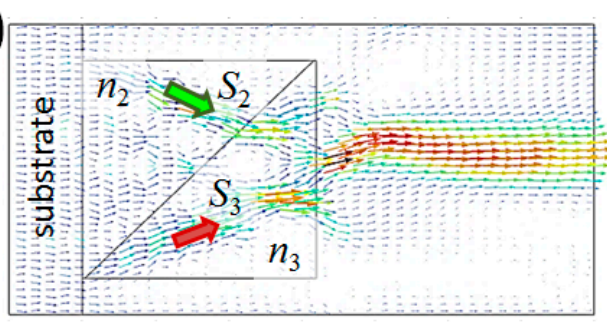

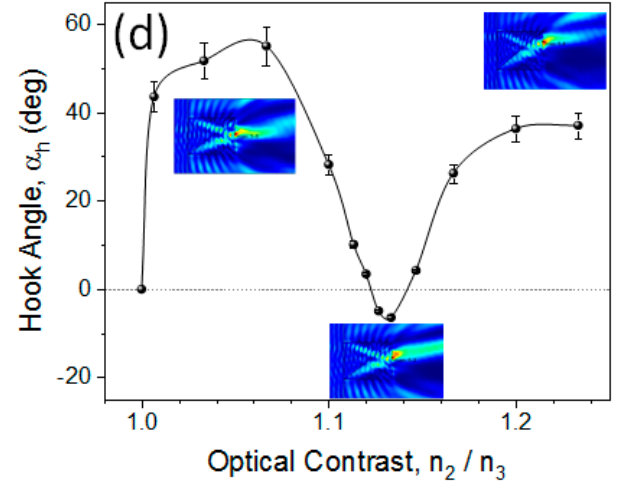

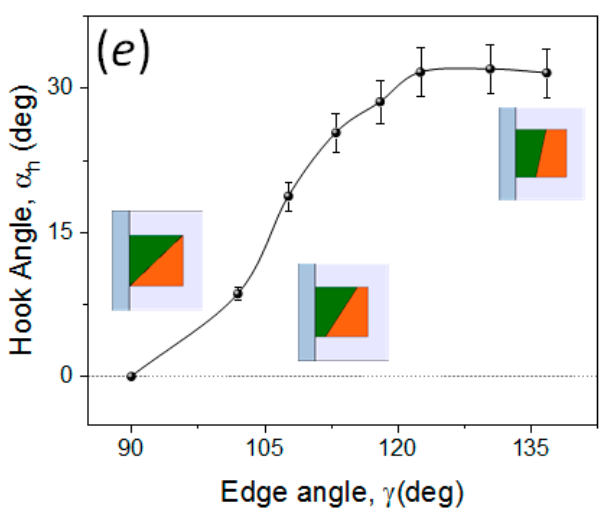

Figure 5. Photonic hook from a Janus slab $\left(L \times H=3.2 \times 3 \lambda^{2}\right)$ exposed to a 500-nm plane wave [59]: (a) geometrical scheme, (b) intensity map and (c) energy fluxes vector map. Hook angle dependence on (d) optical contrast and (e) slab edge angle. 
It is interesting to note the following. As was mentioned above, the main $\mathrm{PH}$ characteristic features are [37,42-46]: (a) the subwavelength radius of curvature, (b) the absence of curved side intensity lobes, and (c) PH emerges near the shadow surface of the mesoscale diffractive optical element. In [60], the authors discussed $\mathrm{PH}$ in the visible band based on ultra-thin metalenses. It must be mentioned that the wave beams considered in [60] do not share the indicative properties of a "photonic hook" and mesoscale conditions, and relate to beams of an intermediate type. This type of beam, structurally speaking, is an Airy-like beam, and not a PH. For example, we directly adopted Figure $1 \mathrm{f}$ from [60], showing the electric field intensity profile under off-axis illumination of the metalens, and processed it as follows. First, this image of intensity spatial distribution was converted to the gray-scale, and then digitized by means of OriginPro 2018 (OriginLab Corporation) data acquisition module. Finally, we picked the horizontal intensity profile at the focal point $\left(y=200\right.$ pixels) and fitted it with the $\operatorname{Ai}(-x)^{2}$ function. The results of this fitting procedure are shown in Figure 6. It is clear that the curved beams considered in [60] resemble closely the classical Airy-type beam [40] (at least up to the 6th side lobe), and cannot be attributed to the PH-type beam, because its field structure possesses a different spatial dependence and physical nature [37,42-46]. In our opinions, the use of the term "photonic hook" in the title and conclusion in [60] strongly misleads the readers.

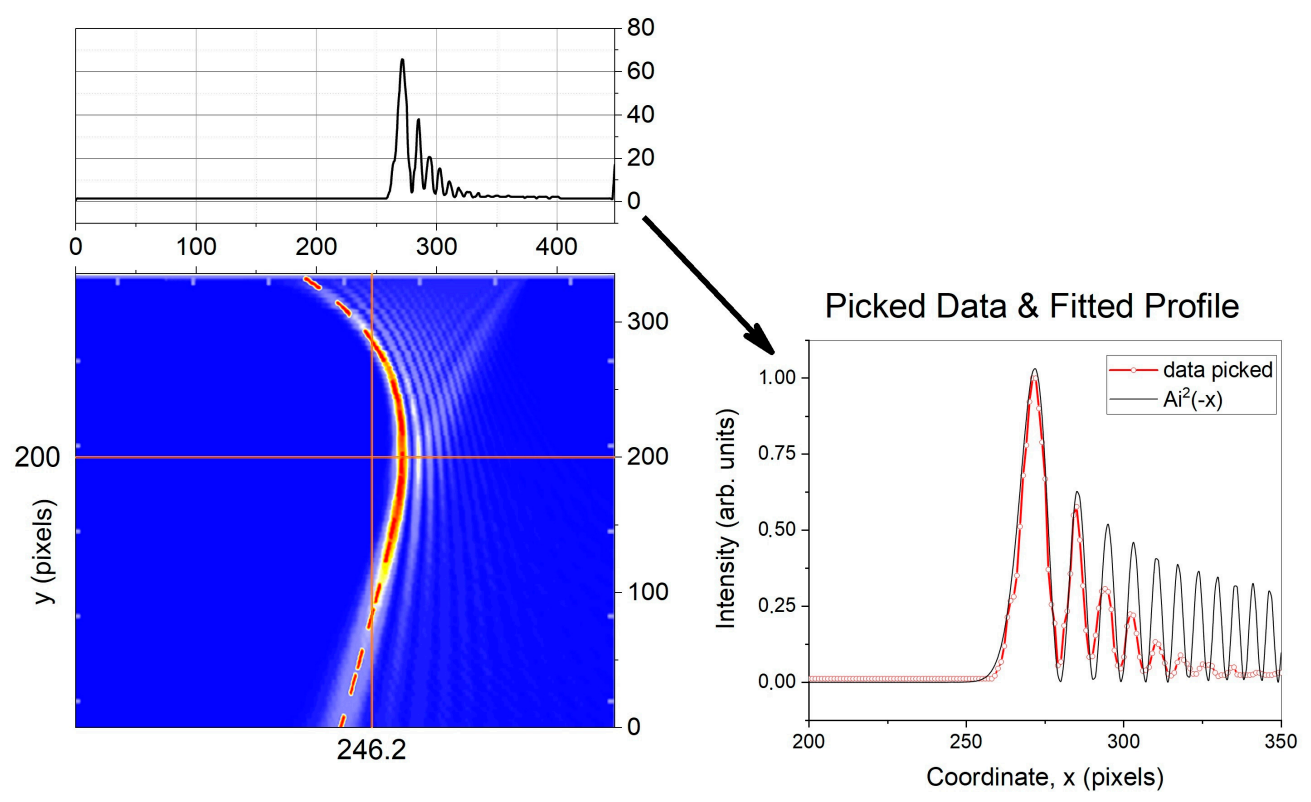

Figure 6. Intensity profile fitting results. Adapted from [58].

\section{Photonic Jet Array from Phase Diffraction Grating}

In Sections 2 and 3, we discuss the single photonic jet and hook generated by a single particle. It was shown above that the diffraction at the phase step and the formation of both curvilinear localized fields and the photonic jet are interconnected. Consider the problem of optical wave diffraction at a 2D phase diffraction grating, from the viewpoint of a PNJ ensemble formation near the grating output facet. In this section, we would like to discuss the photonic jet array generation, because the photonic jet array has more potential applications in practice. In general, the photonic jet array can be obtained by particle array. The regular arrangement of transparent particles is analogous to a phase diffraction grating (PDG). Such periodic structure, with alternating refractive indices, is widely used as a spectral filter or a beam splitter. We present a complete description of the three types of PDGs for generating a photonic jet array. The three most widely used types of dielectric PDGs are the grooves of hemispherical, rectangular and saw-tooth profiles. Figure 7a depicts the schematic diagram of a PDG for a photonic jet, and the dimensions of the PDGs. The length $(\mathrm{L})$ and width $(\mathrm{R})$ are the dimensional parameters of the photonic jet. The focal length $(\mathrm{F})$ and normalized intensity are the key parameters of 
the photonic jet. Laser beams with the wavelengths $405 \mathrm{~nm}, 532 \mathrm{~nm}$ and $671 \mathrm{~nm}$ are incidental from the bottom of the phase diffraction grating. The schematic profiles of gratings are shown in Figure $7 \mathrm{~b}-\mathrm{d}$. All grating profiles are described by the groove distance $d$ and the groove height $h$. The hemispherical and rectangle profiles have a spatial period $\mathrm{P}$. The saw-tooth profile is determined by the blaze angle $\alpha$. In Figure $6 \mathrm{e}-\mathrm{g}$, we show the Finite-Difference Time-Domain (FDTD) simulations of power flow distributions for the PDGs with different groove profiles at a wavelength of $532 \mathrm{~nm}$. The algorithm and details of FDTD simulations were described in references [59,61,62]. According to the physical optics, the mono-layered array of closely packed dielectric particles may be considered as a phase medium with a periodical refractive index [62-64]. The PDGs of different types can be represented as such a medium - the hemispherical grating represents a general-purpose diffraction grating, the rectangle grating provides a method for the application of guided-mode coupling and optical commutation, and the saw-tooth grating has the enhanced capacity for collecting radiation [62-64].

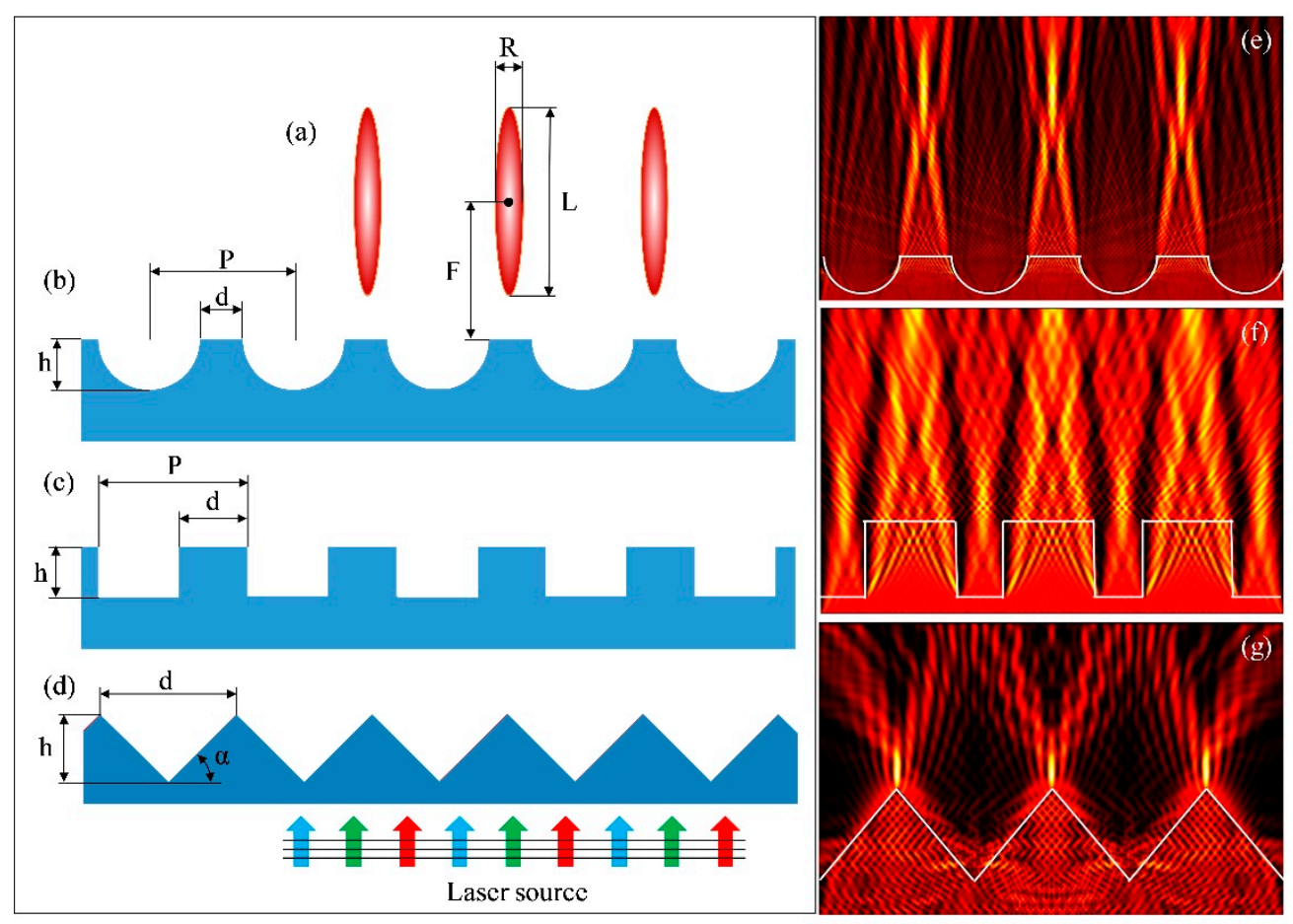

Figure 7. (a) Schematic diagram of a phase diffraction grating for a photonic jet. Schematic profiles of three types of phase diffraction gratings: (b) hemispherical, (c) rectangle, and (d) saw-tooth. FDTD simulation of power flow distributions for the phase diffraction gratings having the grooves of (e) hemispherical, (f) rectangular, and (g) saw-tooth profiles at incident wavelength of $532 \mathrm{~nm}$.

The PDGs were fabricated with a polydimethylsiloxane (PDMS) material. The techniques of lithography and replica molding $[62,65]$ were used primarily to fabricate the phase diffraction gratings. Figure 8 shows the laser scanning digital microscope (LSDM) images of three types of PDGs. The dimensions of the phase diffraction gratings are $\mathrm{d}=5 \mu \mathrm{m}, \mathrm{h}=3.75 \mu \mathrm{m}$ and $p=12.5 \mu \mathrm{m}$ for the hemispherical profile, $\mathrm{d}=5 \mu \mathrm{m}, \mathrm{h}=5 \mu \mathrm{m}$ and $p=7.5 \mu \mathrm{m}$ for the rectangle profile, and $\mathrm{d}=5 \mu \mathrm{m}$, $\mathrm{h}=2.5 \mu \mathrm{m}$ and $\alpha=45$ for the saw-tooth profile. It can be observed that the sidewall of the phase diffraction gratings is almost vertical. Every dimension of the grating profile, from the top of grating to the bottom, is nearly the same. The raw experimental images of power flow distributions for the three types of phase diffraction gratings, with the grooves of hemispherical, rectangular and saw-tooth profiles at incident wavelengths of $405 \mathrm{~nm}, 532 \mathrm{~nm}$ and $671 \mathrm{~nm}$, are shown in Figure 8. The laser beam is incident from the bottom of the grating. The photonic nanojet beam was reconstructed from the collected stack of images by scanning the raw stack of real images along the $\mathrm{z}$ axis for all gratings to get the best small spot. The diffraction gratings of the considered types can form a photonic nanojet array 
with unique characteristics. Rectangular grooves at the wavelength of $405 \mathrm{~nm}$ generate light fluxes with the best spatial localization in the transverse direction (about $0.66 \lambda$ ), with moderate intensity, but the best spatial localization in the transverse direction is generated by the saw-tooth grating at the wavelengths of $532 \mathrm{~nm}$ and $671 \mathrm{~nm}$. The saw-tooth gratings at the designed wavelength generate localized optical fields with the best spatial resolution in the transverse dimensions, and possesses the highest optical intensity. The long photonic nanojet array at the designed wavelength are formed from the gratings with the rectangle groove shape. On the other hand, the greatest focal distance from the grating surface and the long photonic nanojet array are formed by the grating with a hemispherical profile of grooves. The maximal length $(\sim 14.5 \lambda)$ of the photonic nanojet array with the highest optical intensity is observed for the grating with the hemispherical groove at the wavelength of $405 \mathrm{~nm}$.

(a)

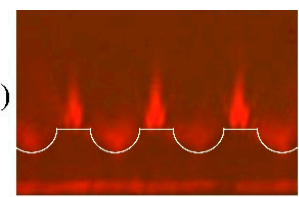

(b)

(c)
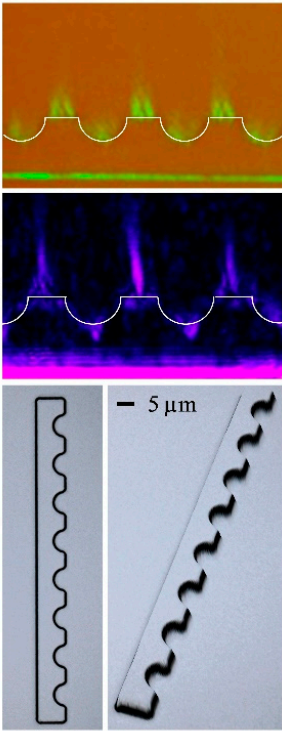

(I)
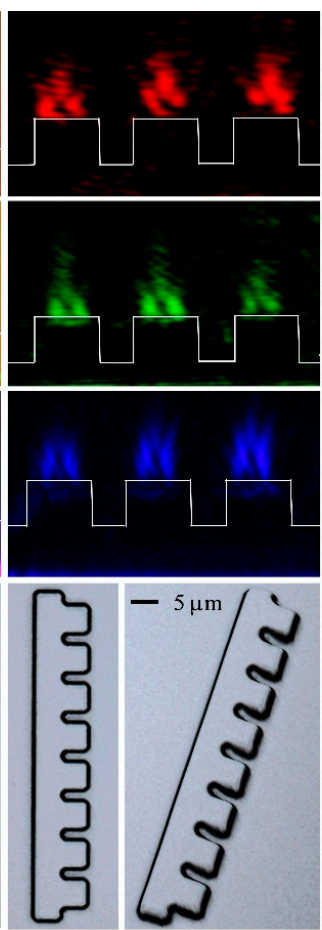

(II)
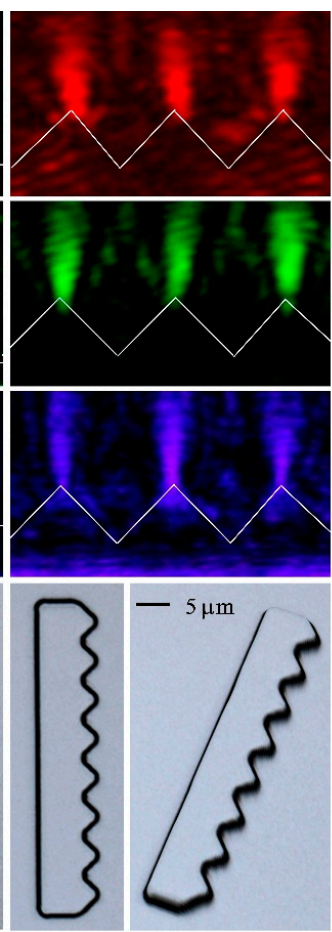

(III)

Figure 8. The raw experimental images of power flow distributions for the three types of phase diffraction gratings having grooves with hemispherical (I), rectangular (II) and saw-tooth (III) profiles, at incident wavelengths (a) $405 \mathrm{~nm}$, (b) $532 \mathrm{~nm}$ and (c) $671 \mathrm{~nm}$. In the bottom, LSDM images of (I) hemispherical, (II) rectangular and (III) saw-tooth phase diffraction gratings; top view (left side) and perspective view (right side) are shown.

A new design of the binary PDG with an embedded pupil opaque mask inside each stripe was proposed in [66]. It was shown that in such a masked phase grating, the spatial resolution of the near-field localization can be brought beyond the solid immersion limit $(\lambda / 2 n)$. Due to an anomalous apodization effect, the subdiffraction field localization is accompanied by an enhancement of intensity, as compared to the classical (non-masked) design. The pupil mask rearranges the optical fluxes within the stripes and promotes the Fano resonance's excitation in the periodic step lattice. The application of this effect in displacement Talbot lithography was discussed in [67].

Figure 9 presents the main photonic jet parameters with the highest quality for each considered PDG type [66]. For the synthetic parameter of PNJ spatial localization, we use the jet quality criterion $Q=\left(I_{\max } L\right) / R[40]$, which combines the three main parameters of a jet ( $I_{\max }$ is peak PNJ intensity). Obviously, the absolute leader in this competition is the grating with rectangular grooves. However, other types of diffraction gratings can be interesting for practical needs, e.g., when the most intensive (hemispherical) or the most remote and elongated PNJs (saw-tooth) are required. It is worth noting 
that the intensity of the photonic jet array for all types of PDG is lower than the typical values for PNJ produced by a spatially localized microsphere [38]. However, the PDG gives more degrees of freedom for manipulating the photonic jet array. The transverse spatial resolution of the PNJ by a PDG is as good as when done with a microspher or microcylinder.

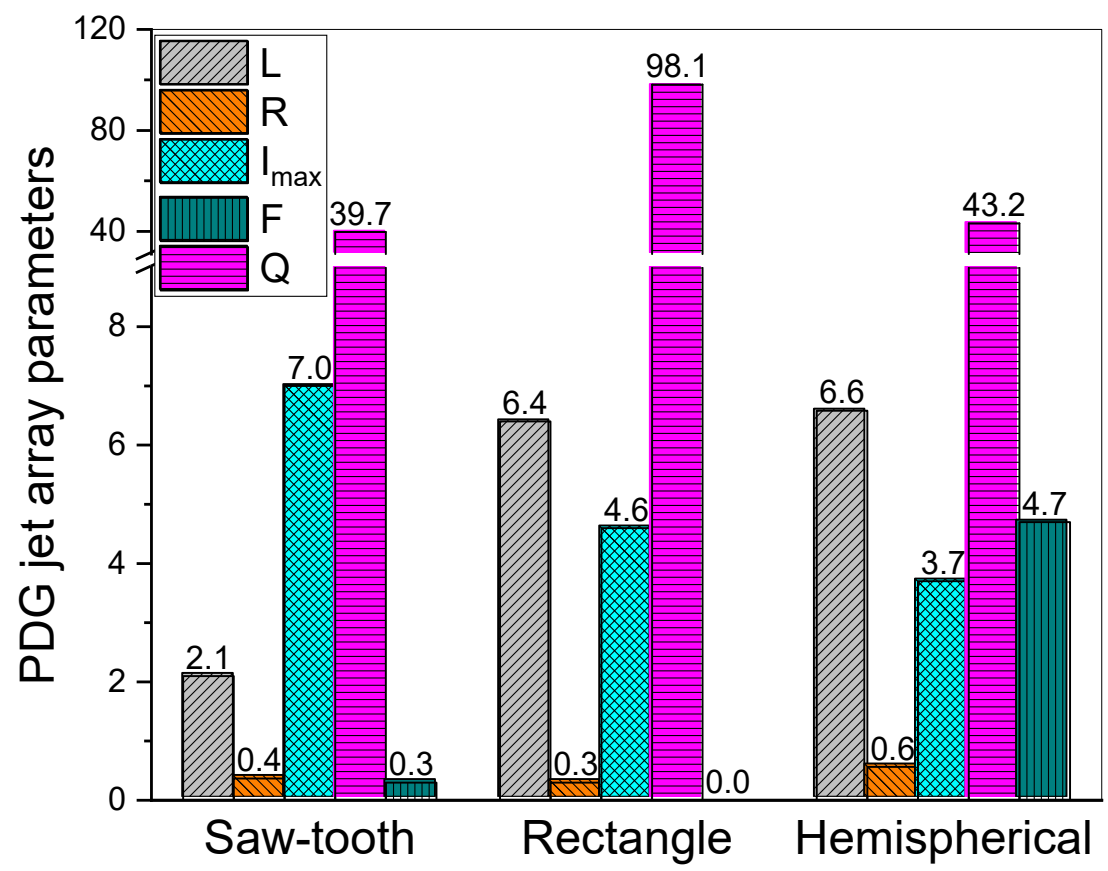

Figure 9. Summary of main PNJ parameters from different PDGs [66].

\section{Conclusions}

Structured dielectric materials that manipulate light in novel ways have many potential applications in the electronic industries and telecommunication. The field of the curve and the array of photonic jets has been the focus of considerable study. Since the first simulation and experimental exposition of a photonic hook in optics, this field has experienced notable growth, making various introductions ranging from new theoretical designs of curved beams, to various generation means and experiments in optics, plasmonic and acoustic. The discussions in this paper include a review of the initial identification of the PNJ for mesoscale particles, the properties of the photonic jet and hook, experimental observations, and the PNJ's array generated by PDGs. The photonic jets demonstrate a unique combination of superior properties, including beam propagation along a curved path, subdiffraction beam width, high peak intensity, and giant backscattering perturbations for nanoscale targets. Accordingly, the photonic jet and hook pose substantial promises with regards to promoting current nano-optics, ranging from optical microscopy, to nanomanufacturing processes and biophotonics. The experimental verification of the concept of the photonic hook, beyond light and into surface waves and acoustics, also merits attention. The photonic hook can be generated by a compact Janus particle, such as a dielectric cuboid, a cylinder with an off-axis Gaussian beam and slab. This simple asymmetric particle functions as an auxiliary structure for subwavelength optical micromanipulation. The nanoscale target trapped by the photonic hook can be transported along a curved trajectory. The behavior of the photonic hook and the optical forces provides new applications in optical tweezing and biological nano-manipulation.

Author Contributions: Conceptualization, idea, O.V.M. and I.V.M.; software, C.-Y.L., O.V.M., I.V.M. and Y.E.G.; formal analysis, O.V.M. and I.V.M.; writing—original draft preparation, O.V.M. and I.V.M.; writing-review and editing, C.-Y.L., Y.E.G., O.V.M. and I.V.M.; visualization, C.-Y.L., Y.E.G., I.V.M.; supervision, O.V.M. and I.V.M.; funding acquisition, O.V.M., I.V.M., Y.E.G. and C.-Y.L. All authors have read and agreed to the published version of the manuscript. 
Funding: This research was funded by Ministry of Science and Technology of Taiwan (MOST 108-2221-E-010-012-MY3, MOST 109-2923-E-010-001-MY2), Yen Tjing Ling Medical Foundation (CI-109-24), Russian Foundation for Basic Research (20-57-S52001), Ministry of Science and Higher Education of the Russian Federation and partially carried out within the framework of the Tomsk Polytechnic and Tomsk State Universities Competitiveness Enhancement Programs, Russia.

Conflicts of Interest: The authors declare no conflict of interest.

\section{References}

1. Berry, M.V.; Balazs, N.L. Nonspreading wave packets. Am. J. Phys. 1979, 47, 264-267. [CrossRef]

2. Siviloglou, G.A.; Broky, J.; Dogariu, A.; Christodoulides, D.N. Observation of accelerating Airy beams. Phys. Rev. Lett. 2007, 99, 213901. [CrossRef] [PubMed]

3. Greenfield, E.; Segev, M.; Walasik, W.; Raz, O. Accelerating light beams along arbitrary convex trajectories. Phys. Rev. Lett. 2011, 106, 213902. [CrossRef] [PubMed]

4. Kaminer, I.; Bekenstein, R.; Nemirovsky, J.; Segev, M. Nondiffracting accelerating wave packets of Maxwell's equations. Phys. Rev. Lett. 2012, 108, 163901. [CrossRef] [PubMed]

5. Zhang, P.; Hu, Y.; Li, T.; Cannan, D.; Yin, X.; Morandotti, R.; Chen, Z.; Zhang, X. Nonparaxial Mathieu and Weber accelerating beams. Phys. Rev. Lett. 2012, 109, 193901. [CrossRef]

6. Aleahmad, P.; Miri, M.A.; Mills, M.S.; Kaminer, I.; Segev, M.; Christodoulides, D.N. Fully vectorial accelerating diffraction-free Helmholtz beams. Phys. Rev. Lett. 2012, 109, 203902. [CrossRef]

7. Baumgartl, J.; Mazilu, M.; Dholakia, K. Optically mediated particle clearing using Airy wavepackets. Nat. Photonics 2008, 2, 675-678. [CrossRef]

8. Polynkin, P.; Koleskik, M.; Moloney, J.V.; Siviloglou, G.A.; Christodoulides, D.N. Curved plasma channel generation using ultraintense Airy beams. Science 2009, 324, 229-232. [CrossRef]

9. Lin, J.; Dellinger, J.; Genevet, P.; Cluzel, B.; de Fornel, F.; Capasso, F. Cosine-gauss plasmon beam: A localized long-range nondiffracting surface wave. Phys. Rev. Lett. 2012, 109, 093904. [CrossRef]

10. Li, L.; Li, T.; Wang, S.M.; Zhu, S.N. Collimated plasmon beam: Nondiffracting versus linearly focused. Phys. Rev. Lett. 2013, 110, 046807. [CrossRef]

11. Ellenbogen, T.; Voloch-Bloch, N.; Ganany-Padowicz, A.; Arie, A. Nonlinear generation and manipulation of Airy beams. Nat. Photonics 2009, 3, 395-398. [CrossRef]

12. Voloch-Bloch, N.; Lereah, Y.; Lilach, Y.; Gover, A.; Arie, A. Generation of electron Airy beams. Nature 2013, 494, 331-335. [CrossRef] [PubMed]

13. Efremidis, N.K.; Chen, Z.; Segev, M.; Christodoulides, D.N. Airy beams and accelerating waves: An overview of recent advances. Optica 2019, 6, 686-701. [CrossRef]

14. Froehl, L.; Froehly, L.; Courvoisier, F.; Mathis, A.; Jacquot, M.; Furfaro, L.; Giust, R.; Lacourt, P.A.; Dudley, J.M. Arbitrary accelerating micron-scale caustic beams in two and three dimensions. Opt. Express 2011, 19, 16455-16465. [CrossRef] [PubMed]

15. Gao, N.; Li, H.; Zhu, X.; Hua, Y.; Xie, C. Quasi-periodic gratings: Diffraction orders accelerate along curves. Opt. Lett. 2013, 38, 2829-2831. [CrossRef]

16. He, J.; Wang, S.; Xie, Z.; Ye, J.; Wang, X.; Kan, Q.; Zhang, Y. Abruptly autofocusing terahertz waves with meta-hologram. Opt. Lett. 2016, 41, 2787-2790. [CrossRef]

17. Kondakci, H.E.; Abouraddy, A.F. Airy wave packets accelerating in space-time. Phys. Rev. Lett. 2018, 120, 163901. [CrossRef]

18. Durnin, J.; Miceli, J.J.; Eberly, J.H. Diffraction-free beams. Phys. Rev. Lett. 1987, 58, 1499-1501. [CrossRef]

19. Matijošius, A.; Jarutis, V.; Piskarskas, A. Generation and control of the spiraling zero-order Bessel beam. Opt. Express 2010, 18, 8767-8771. [CrossRef]

20. Morris, J.; Cizmar, T.; Dalgarno, H.; Marchington, R.; Gunn-Moore, F.; Dholakia, K. Realization of curved Bessel beams: Propagation around obstructions. J. Opt. 2010, 12, 124002. [CrossRef]

21. Chremmos, I.D.; Chen, Z.; Christodoulides, D.N.; Efremidis, N.K. Bessel-like optical beams with arbitrary trajectories. Opt. Lett. 2012, 37, 5003-5005. [CrossRef] [PubMed]

22. Zhao, J.; Zhang, P.; Deng, D.; Liu, J.; Gao, Y.; Chremmos, I.D.; Efremidis, N.K.; Christodoulides, D.N.; Chen, Z Observation of selfaccelerating Bessel-like optical beams along arbitrary trajectories. Opt. Lett. 2013, 38, 498-500. [CrossRef] [PubMed] 
23. Zhao, J.; Chremmos, I.D.; Song, D.; Christodoulides, D.N.; Efremidis, N.K.; Chen, Z. Curved singular beams for three-dimensional particle manipulation. Sci. Rep. 2015, 5, 12086. [CrossRef] [PubMed]

24. Vettenburg, T.; Dalgarno, H.; Nylk, J.; Coll-Lladó, C.; Ferrier, D.; Čižmár, T.; Gunn-Moore, F.; Dholakia, K. Light-sheet microscopy using an Airy beam. Nat. Methods 2014, 11, 541-544. [CrossRef]

25. Domenico, G.; Ruocco, G.; Colosi, C.; DelRe, E.; Antonacci, G. Cancellation of Bessel beam side lobes for high-contrast light sheet microscopy. Sci. Rep. 2018, 8, 17178. [CrossRef] [PubMed]

26. Chan, W.L.; Chen, H.; Taylor, A.J.; Brener, I.; Cich, M.J.; Mittleman, D.M. A spatial light modulator for terahertz beams. Appl. Phys. Lett. 2009, 94, 213511. [CrossRef]

27. Valdmann, A.; Piksarv, P.; Valtna-Lukner, H.; Saari, P. White-light hyperbolic Airy beams. J. Opt. 2018, $20,095605$. [CrossRef]

28. Kopylov, Y.V.; Popov, A.V. Diffraction phenomena inside thick Fresnel zone plates. Radio Sci. 1996, 31, 1815-1822. [CrossRef]

29. Born, M.; Wolf, E. Principles of Optics, 6th ed.; Pergamon: Oxford, UK, 1986.

30. Pacheco-Peña, V.; Beruete, M.; Minin, I.V.; Minin, O.V. Terajets produced by dielectric cuboids. Appl. Phys. Lett. 2014, 105, 084102. [CrossRef]

31. Minin, O.V.; Minin, I.V. Terahertz artificial dielectric cuboid lens on substrate for super-resolution images. Opt. Quantum Electron. 2017, 49, 326-329. [CrossRef]

32. Heifetz, A.; Kong, S.; Sahakian, A.; Taflove, A.; Backman, V. Photonic nanojets. J. Comput. Theor. Nanosci. 2009, 6, 1979-1992. [CrossRef]

33. Liu, C.; Wang, Y. Real-space observation of photonic nanojet in dielectric microspheres. Physica E 2014, 61, 141-147. [CrossRef]

34. Luk'yanchuk, B.; Paniagua-Domínguez, R.; Minin, I.V.; Minin, O.V.; Wang, Z. Refractive index less than two: Photonic nanojets yesterday, today and tomorrow. Opt. Mater. Express 2017, 7, 1820-1847. [CrossRef]

35. Arya, A.; Laha, R.; Das, G.M.; Dantham, V.R. Enhancement of Raman scattering signal using photonic nanojet of portable and reusable single microstructures. J. Raman Spectrosc. 2018, 49, 897-902. [CrossRef]

36. Zhang, X.; Chen, I.; Chang, C. Recent progress in near-field nanolithography using light interactions with colloidal particles: From nanospheres to three-dimensional nanostructures. Nanotechnology 2019, 30, 352002. [CrossRef] [PubMed]

37. Minin, I.V.; Minin, O.V. Diffractive Optics and Nanophotonics: Resolution below the Diffraction Limit; Springer: Berlin, Germany, 2016.

38. Geints, Y.; Panina, E.K.; Zemlyanov, A.A. Control over parameters of photonic nanojets of dielectric microspheres. Opt. Commun. 2010, 283, 4775-4781. [CrossRef]

39. Cao, Y.; Liu, Z.; Minin, O.V.; Minin, I.V. Deep subwavelength-scale light focusing and confinement in nanohole-structured mesoscale dielectric spheres. Nanomaterials 2019, 9, 186. [CrossRef]

40. Wang, Z.; Luk'yanchuk, B.; Yue, L.; Paniagua-Domínguez, R.; Yan, B.; Monks, J.; Minin, O.V.; Minin, I.V.; Huang, S.; Fedyanin, A. High order Fano resonances and giant magnetic fields in dielectric microspheres. Sci. Rep. 2019, 9, 20293. [CrossRef]

41. Yue, L.; Yan, B.; Monks, J.; Dhama, R.; Jiang, C.; Minin, O.V.; Minin, I.V.; Wang, Z. Full three-dimensional Poynting vector analysis of great field-intensity enhancement in a specifically sized spherical-particle. Sci. Rep. 2019, 9, 20224. [CrossRef]

42. Dholakia, K.; Bruce, G. Optical hooks. Nat. Photonics 2019, 13, 229-230. [CrossRef]

43. Yue, L.; Minin, O.V.; Wang, Z.; Monks, J.; Shalin, A.; Minin, I.V. Photonic hook: A new curved light beam. Opt. Lett. 2018, 43, 771-774. [CrossRef]

44. Ang, A.; Karabchevsky, A.; Minin, I.V.; Minin, O.V.; Sukhov, S.; Shalin, A. Photonic Hook based optomechanical nanoparticle manipulator. Sci. Rep. 2018, 8, 2029. [CrossRef] [PubMed]

45. Spector, M.; Ang, A.; Minin, O.V.; Minin, I.V.; Karabchevsky, A. Temperature mediated 'Photonic hook' nanoparticle manipulator with pulsed illumination. Nanoscale Adv. 2020. [CrossRef]

46. Minin, I.V.; Minin, I.V.; Katyba, G.; Chernomyrdin, N.; Kurlov, V.; Zaytsev, K.; Yue, L.; Wang, Z.; Christodoulides, D. Experimental observation of a photonic hook. Appl. Phys. Lett. 2019, 114, 031105. [CrossRef]

47. Rubio, C.; Tarrazó-Serrano, D.; Minin, O.V.; Uris, A.; Minin, I.V. Acoustical hooks: A new subwavelength self-bending beam. Results Phys. 2020, 16, 102921. [CrossRef] 
48. Minin, I.V.; Minin, O.V.; Ponomarev, D.S.; Glinskiy, I.A. Photonic hook plasmons: A new curved surface wave. Ann. Phys. 2018, 530, 1800359. [CrossRef]

49. Minin, I.V.; Minin, O.V.; Glinskiy, I.A.; Khabibullin, R.A.; Malureanu, R.; Yakubovsky, D.I.; Volkov, V.; Ponomarev, D.S. Experimental verification of a plasmonic hook in a dielectric Janus particle. arXiv 2020, arXiv:2004.10749.

50. Yang, J.; Twardowski, P.; Gérard, P.; Duo, Y.; Fontaine, J.; Lecler, S. Ultra-narrow photonic nanojets through a glass cuboid embedded in a dielectric cylinder. Opt. Express 2018, 26, 3723-3731. [CrossRef]

51. Xing, E.; Gao, H.; Rong, J.; Khew, S.; Liu, H.; Tong, C.; Hong, M. Dynamically tunable multi-lobe laser generation via multifocal curved beam. Opt. Express 2018, 26, 30944-30951. [CrossRef]

52. Minin, I.V.; Minin, O.V.; Liu, C.-Y.; Wei, H.-D.; Karabchevsky, A. Simulation and experimental observation of tunable photonic nanojet and photonic hook upon asymmetric illumination of a mesoscale cylinder with mask. arXiv 2020, arXiv:2004.05911.

53. Huang, Y.; Zhen, Z.; Shen, Y.; Min, C.; Veronis, G. Optimization of photonic nanojets generated by multilayer microcylinders with a genetic algorithm. Opt. Express 2019, 27, 1310-1325. [CrossRef] [PubMed]

54. Minin, I.V.; Minin, O.V. Subwavelength Self-Bending Structured Light Beams. Carbon Nanostructures and their Electromagnetic Properties. In Proceedings of the Fourth Russian-Belarusian Workshop, Tomsk, Russia, 21-24 April 2019; pp. 52-57.

55. Minin, I.V.; Minin, O.V. Dielectric particle-based strategy to design a new self-bending subwavelength structured light beams. In Proceedings of the 14th International Forum on Strategic Technology, Tomsk, Russia, 14-17 October 2019; p. 23.

56. Gu, G.; Shao, L.; Song, J.; Qu, J.; Zheng, K.; Shen, X.; Peng, Z.; Hu, J.; Chen, X.; Chen, M.; et al. Photonic hooks from Janus microcylinders. Opt. Express 2019, 27, 37771-37780. [CrossRef] [PubMed]

57. Shen, X.; Gu, G.; Shao, L.; Peng, Z.; Hu, J.; Bandyopadhyay, S.; Liu, Y.; Jiang, J.; Chen, M. Twin photonic hook generated by twin-ellipse microcylinder. IEEE Photonics J. 2020, 12. [CrossRef]

58. Ang, A.S.; Minin, I.V.; Minin, O.V.; Sukhov, S.V.; Shalin, A.; Karabchevsky, A. Low-Contrast Photonic Hook Manipulator for Cellular Differentiation. In Proceedings of the 9th International Conference on Metamaterials, Photonic Crystals and Plasmonics, Marseille, France, 24 June-1 July 2018.

59. Geints, Y.E.; Minin, I.V.; Minin, O.V. Tailoring "photonic hook" from Janus dielectric microbar. J. Opt. 2020, 22, 065606. [CrossRef]

60. Ma, X.; Guo, Y.; Pu, M.; Jin, J.J.; Gao, P.; Li, X.; Luo, X. Tunable optical hooks in the visible band based on ultra-thin metalenses. Ann. Phys. 2020, 532, 1900396. [CrossRef]

61. Taflove, A.; Hagness, S. Computational Electrodynamics: The Finite Difference Time Domain Method; Artech House: Norwood, MA, USA, 1998.

62. Geints, Y.; Zemlyanov, A. Modeling spatially localized photonic nanojets from phase diffraction gratings. J. Appl. Phys. 2016, 119, 153101. [CrossRef]

63. Liu, C.; Minin, O.V.; Minin, I.V. First experimental observation of array of photonic jets from saw-tooth phase diffraction grating. EPL 2018, 123, 54003. [CrossRef]

64. Liu, C.; Yen, T.; Minin, O.V.; Minin, I.V. Theoretical and experimental investigations of photonic jet array from rectangle phase diffraction grating. In Proceedings of the SPIE 10712, Optical Manipulation Conference, 107121H, Yokohama, Japan, 24 April 2018.

65. Zhang, Y.; Lo, C.; Taylor, J.; Yang, S. Replica molding of high-aspect-ratio polymeric nanopillar arrays with high fidelity. Langmuir 2006, 22, 8595-8601. [CrossRef]

66. Geints, Y.; Minin, O.V.; Minin, I.V. Apodization-Assisted subdiffraction near-field focusing in 2D phase diffraction grating. Ann. Phys. 2019, 531, 1900033. [CrossRef]

67. Geints, Y.; Minin, O.V.; Minin, I.V.; Zemlyanov, A. Self-images contrast enhancement for displacement Talbot lithography by means of composite mesoscale amplitude-phase masks. J. Opt. 2019, 22, 015002. [CrossRef]

(C) 2020 by the authors. Licensee MDPI, Basel, Switzerland. This article is an open access article distributed under the terms and conditions of the Creative Commons Attribution (CC BY) license (http://creativecommons.org/licenses/by/4.0/). 